\title{
Dinâmica da regeneração do subsolo de áreas degradadas dentro do bioma Cerrado
}

\author{
$\overline{\text { Graciela B. Rodrigues }^{1} \text {, Kátia L. Maltoni², Ana Maria R. Cassiolato }}{ }^{2}$
}

\begin{abstract}
RESUMO
Realizou-se este trabalho com o objetivo de se avaliar as modificações ocorridas nas propriedades físicas e químicas do subsolo revegetado, em áreas degradadas dentro do bioma Cerrado e verificar qual das coberturas vegetais estudadas promoveu melhores condições para a recuperação do solo. A pesquisa foi conduzida em áreas degradadas remanescentes da construção da Usina Hidrelétrica de Ilha Solteira, em Ilha Solteira (SP). O delineamento utilizado foi inteiramente casualizado com cinco tratamentos e três repetições. Avaliaram-se: áreas em regeneração natural, áreas com braquiária, com Pinus sp., com solo exposto e cerrado (utilizado como controle). Foram avaliados: porosidade do solo, densidade, estabilidade de agregados, taxa de infiltração, temperatura, cátions trocáveis, matéria orgânica, pH e acidez potencial. Os resultados mostraram que o Pinus não é uma boa espécie para recuperar os atributos químicos do subsolo. As áreas revegetadas precisam ser melhoradas em seus atributos físicos, para um adequado desenvolvimento da vegetação. A braquiária e a regeneração foram os tratamentos que apresentaram os melhores resultados, aproximando-se mais do Cerrado natural.
\end{abstract}

Palavras-chave: propriedades físicas do solo, fertilidade do solo, Pinus, braquiária, savana

\section{Dynamics of the subsoil regeneration in degraded areas of Cerrado}

\begin{abstract}
The objective of this research was to evaluate the physical and chemical conditions of revegetated subsoil in degraded areas in a Cerrado biome and to verify which plants promote better conditions for soil recovery. The research was conducted in the remaining area of the hydroelectrical plant site at Ilha Solteira (SP). The experimental design was a completely randomized with five treatments and three replications. The treatments consisted of: natural regeneration area, brachiaria area, Pinus sp. area, exposed soil area and Cerrado grassland (used as control). The following soil characteristics were appraised: porosity; density, stability of aggregates, infiltration rate, temperature, exchangeable cations, organic matter, $\mathrm{pH}$ and potential acidity. The results show that Pinus is not a good species to recover the chemical attributes of the subsoil. The revegetated areas need to be improved in their physical attributes to allow a better development of the vegetation. Brachiaria and the natural regeneration were the most promising treatments, presenting results similar to natural Cerrado.
\end{abstract}

Key words: physical soil properties, soil fertility, Pinus, brachiaria, savanna 


\section{INTRODUÇÃO}

Áreas degradadas referem-se a ecossistemas alterados, onde perdas ou excessos são as formas mais comuns de perturbações e degradações ambientais. A retirada da cobertura vegetal, dependendo da intensidade, pode ser considerada uma degradação ou uma perturbação ambiental. Caso o ambiente não se recupere, diz-se que está degradado e necessita de intervenções mas, se mantém sua capacidade de regeneração, diz-se que o ambiente está perturbado e intervenções poderão acelerar o processo de recuperação (Corrêa \& Melo, 1998).

Ante esta definição, no entorno da Usina Hidrelétrica de Ilha Solteira, as “áreas de empréstimo” podem ser consideradas áreas degradadas, pois delas foram retirados os horizontes superficiais do solo; são áreas com intensos sinais de degradação que, apesar de não sofrerem intervenções há 30 anos, apresentam insignificante regeneração natural, mesmo estando dentro do bioma cerrado que, de acordo com Durigan (1999), nessas condições, degradação por terraplenagem (cortes), apresentam potencial de regeneração natural médio e lento.

O processo de regeneração natural se inicia com a reprodução de espécies, cujas sementes ali chegam, germinam e se estabelecem. Características do local como textura, instabilidade da superfície, compactação, retenção de água, pH, distância do banco de sementes, dentre outros, determinam as espécies adaptadas a se estabelecerem no local (Almeida, 2002).

Os principais problemas edáficos encontrados em áreas de empréstimo ou mineradas, são a compactação do material exposto, as baixas taxas de infiltração e capacidade de armazenamento de água, a deficiência de oxigênio, a alta resistência à penetração de raízes, o aumento da densidade do solo e a falta de matéria orgânica (Moreira, 2004).

Em áreas degradadas pela remoção da vegetação e do solo ou de parte de suas camadas e se levando à exposição do horizonte $\mathrm{C}$ ou, ainda, do subsolo, tem-se buscado a recuperação por meio da revegetação, pois é de amplo conhecimento a inter-relação da vegetação com a morfologia, a química e a biologia do solo (Resende et al., 2002; Garay et al., 2003), além de melhorar, segundo Lyle (1987), a estética visual da área.

É importante se conhecer, neste contexto, os atributos do solo e da vegetação que possam interferir no processo de degradação do ambiente (Resende et al., 2002). Do solo são pertinentes: porosidade, espessura do horizonte A, profundidade do sólum, textura, capacidade de retenção de água, estrutura, suprimento de nutrientes e $\mathrm{pH}$, entre outras e, da vegetação, o tipo e intensidade de cobertura (Doran \& Parkin, 1994; Resende et al., 2002).

Sabe-se que as características físicas do solo têm a responsabilidade de transportar ar, calor, água e substâncias solúveis e, em seu estado natural (sob vegetação natural) os solos se encontram em equilíbrio com o meio, e que os Latossolos, em particular, possuem excelentes propriedades físicas nessas condições (Sánchez, 1981).

A cobertura vegetal atua como elemento responsável pela estruturação do solo através do sistema radicular. O siste- ma radicular das matas, por sua vez, constitui-se em uma malha densa de raízes que confere, à porção superficial do solo, uma defesa eficiente contra a ação erosiva da água, defesa esta que se manifesta na forma de resistência a erosão e aumenta proporcionalmente, com a densidade das raízes (Prandini et al., 1982).

A qualidade do sistema solo é definida por suas funções, que representam uma combinação de suas propriedades físicas, químicas e biológicas, as quais, por sua vez, provêm um meio para o crescimento de plantas, regulam o fluxo de água no ambiente e servem como tampão na formação, atenuação e degradação de compostos nocivos ao ambiente, pois sustentam uma enorme população de microrganismos (Doran \& Parkin, 1994; Sposito, 1998).

Ressalta-se que são raras as pesquisas que procuram avaliar a qualidade do solo sob o enfoque de degradação; certamente é uma área por meio da qual as pesquisas precisam evoluir para que rotinas sejam estabelecidas e o monitoramento e diagnóstico sejam facilmente realizáveis, pois a reabilitação de áreas degradadas não consiste de ações isoladas mas, sim, de um conjunto de atividades que têm, por objetivo, recompor a paisagem que foi perturbada (Dias Filho, 1998; Dias \& Griffith, 1998).

Dentro deste contexto, ralizou-se o presente trabalho com o objetivo de se avaliar as modificações ocorridas nas propriedades físicas e químicas do subsolo revegetado, em áreas sob influência da Usina Hidrelétrica de Ilha Solteira, no Município de Selvíri, MS, e determinar qual das coberturas vegetais estudadas promoveu melhores condições para o desenvolvimento do solo.

\section{MATERIAL E MÉTODOS}

A área selecionada para estudo fica à jusante da Usina Hidrelétrica de Ilha Solteira, no município de Selvíria, MS, apresenta latitude e longitude de $20^{\circ} 22^{\prime}$ e $51^{\circ} 24^{\prime}$, respectivamente. A altitude média no local é de $335 \mathrm{~m}$, com clima do tipo Aw (tropical úmido com estação chuvosa no verão e seca no inverno), segundo o sistema internacional de Köeppen (19451962). A temperatura média anual é de $23,7^{\circ} \mathrm{C}$ e a precipitação média anual é de 1300 mm (Demattê, 1980).

Os solos mais representativos na região são Latossolos Vermelhos e Argissolos Vermelhos (Demattê, 1980; EMBRAPA, 1999; Moniz \& Carvalho, 1973). Os materiais de origem desses solos são sedimentos do Arenito Bauru associados, em alguns locais, a material retrabalhado de basalto, da Formação Serra Geral (Moniz \& Carvalho, 1973; IPT, 1981) gerando nas áreas um subsolo argilo-arenoso.

Em 1995 a área em estudo foi utilizada como "área de empréstimo”, isto é, área de onde se removeu material para a construção da Usina Hidrelétrica de Ilha Solteira. Nessas áreas, a vegetação foi retirada e camadas de solo e subsolo removidas, algumas das quais em $10 \mathrm{~m}$ de espessura em alguns pontos; posteriormente, parte dessa "área de empréstimo" foi revegetada com Pinus sp. (1984) e outra foi ocupada espontaneamente por Brachiaria brizantha; do restante, uma parte está em processo de regeneração natural, com espécies nativas de 
Cerrado, e o que sobrou permanece com o subsolo exposto não apresentando indícios de regeneração natural, mesmo após 30 anos de abandono.

Para a execução do trabalho alocaram-se três parcelas de $200 \mathrm{~m}^{2}$ em cada uma das situações citadas, com localização em campo aleaória, dentro de cada área. Para instalação das parcelas em campo foram considerados os mesmos solo/subsolo, relevo e clima. Os tratamentos considerados foram: Cerrado - área com vegetação e solo preservados; regeneração - área de empréstimo em processo de regeneração natural com espécies de Cerrado; braquiária - área de empréstimo espontaneamente ocupada por Brachiaria brizantha; Pinus - área de empréstimo reflorestada com Pinus sp., em 1984 e solo exposto - área de empréstimo abandonada, onde o subsolo não foi revegetado e não se observa a ocorrência de processo de regeneração natural.

Nas análises químicas do material avaliaram-se três repetições por tratamento (coberturas vegetais), sendo cada repetição constituída da média aritmética dos resultados da análise de quatro amostras compostas, oriundas de 10 amostras simples do material de cada parcela, nas profundidades de $0-0,05,0,05-0,10,0,10-0,20,0,20-0,40$ e 0,40-0,60 m. As análises realizadas foram: cátions trocáveis, extração feita com resina trocadora de íons; capacidade de troca catiônica (CTC), obtida por meio da soma de bases e acidez potencial $\left(\mathrm{H}^{+}+\mathrm{Al}^{+3}\right)$; fósforo disponível, extração feita com resina trocadora; matéria orgânica (MO), método colorimétrico; alumínio trocável, extração com $\mathrm{KCl}$ e pH em $\mathrm{CaCl}_{2}$ (van Raij \& Quaggio, 1983).

Para as análises físicas do material seguiu-se a particularidade de cada metodologia, procurando-se, sempre que possível, adaptá-las às profundidades de $0-0,10,0,10-0,20$, 0,20-0,40 e 0,40-0,60 m. As análises foram realizadas de acordo com EMBRAPA (1997), considerando-se três repetições por cobertura vegetal e por profundidade, como segue: densidade do solo, método do anel volumétrico; densidade de partículas, método do balão volumétrico e álcool etílico como líquido penetrante; macroporosidade e microporosidade, método da "mesa de tensão", com o emprego de amostra com estrutura não deformada; estabilidade de agregados em água para as profundidades de 0-0,10 e 0,10-0,20 m e velocidade de infiltração, método de campo, com auxílio do infiltrômetro. A temperatura do solo foi coletada com termômetro de mão, a 0,04 $\mathrm{m}$ de profundidade; efetuaram-se 10 leituras por parcela, nos meses de março, junho, setembro e dezembro, às 8:00 e às 16:00 h (Doran \& Parkin, 1994).

O delineamento experimental utilizado foi o casualizado, sendo os dados submetidos à análise de variância, e as médias comparadas pelo teste de Tukey a 5\% de probabilidade. As análises estatísticas foram realizadas por meio do programa SAS (SAS Institute, 1999).

\section{RESULTADOS E DISCUSSÃO}

\section{Atributos químicos}

Diferenças significativas formam verificadas, para todas as variáveis, entre as coberturas vegetais e entre profundidades dentro da mesma área (exceções para pH e Al) (Tabela 1). Interações significativas foram verificadas entre coberturas vegetais e profundidades para as variáveis $\mathrm{P}, \mathrm{MO}$, $\mathrm{H}+\mathrm{Al}$, Al e CTC (Tabela 2).

O comportamento da CTC acompanha o constatado para MO e indica o Pinus como o pior tratamento, pois apresenta a menor CTC em todas as profundidades (Tabela 2). Os melhores resultados para CTC foram encontrados na regeneração e na braquiária, que mais se aproximaram do cerrado, na menor profundidade.

Este comportamento da CTC, semelhante ao da MO, reforça a estreita ligação entre essas variáveis, como também relatam Garay et al. (2003) para solos cultivados com Acacia mangium e Eucalyptus grandis. A partir de 0,20 m de profundidade não se verifica diferença significativa da CTC entre as coberturas vegetais, exceto para o Pinus, sugerindo

Tabela 1. Médias* e valores de $\mathrm{F}$ para as variáveis fósforo $(\mathrm{P})$, matéria orgânica $(\mathrm{MO})$, potásssio $\left(\mathrm{K}^{+}\right)$, cálcio $\left(\mathrm{Ca}^{++}\right)$, magnésio $\left(\mathrm{Mg}^{++}\right)$, acidez potencial $(\mathrm{H}+\mathrm{Al})$, alumínio trocável $\left(\mathrm{Al}{ }^{+++}\right)$, soma de bases $(\mathrm{SB})$, capacidade de trocas catiônicas $(\mathrm{CTC})$ e saturação por bases $(\mathrm{V})$, nas diferentes coberturas vegetais (CVeg) e profundidades (Prof)

\begin{tabular}{|c|c|c|c|c|c|c|c|c|c|c|c|c|}
\hline \multirow{2}{*}{ Variáveis } & \multirow{2}{*}{ Tratamentos } & \multirow{2}{*}{$\underset{\mathrm{CaCl}_{2}}{\mathrm{pH}}$} & \multirow{2}{*}{$\begin{array}{l}\text { P resina } \\
\left(\mathrm{mg} \mathrm{dm}^{-3}\right)\end{array}$} & \multirow{2}{*}{$\begin{array}{c}\mathrm{MO} \\
\left(\mathrm{g} \mathrm{dm}^{-3}\right)\end{array}$} & K & $\mathrm{Ca}$ & Mg & $\mathrm{H}+\mathrm{Al}$ & Al & SB & CTC & \multirow{2}{*}{$\begin{array}{l}\mathbf{V} \\
\%\end{array}$} \\
\hline & & & & & \multicolumn{7}{|c|}{$\mathrm{mml}_{\mathrm{c}} \mathrm{dm}^{-3}$} & \\
\hline \multirow[t]{5}{*}{ CVeg } & Cerrado & $5,3 a b$ & $5,4 a$ & $19,7 b$ & $0,8 a$ & $6,7 b$ & $3,5 b c$ & $44,1 \mathrm{a}$ & $9,1 \mathrm{a}$ & $9,7 \mathrm{bc}$ & $53,8 a$ & $17,1 \mathrm{~b}$ \\
\hline & Regeneração & $5,2 \mathrm{ab}$ & $3,5 b$ & $25,2 a$ & $1,0 \mathrm{a}$ & $9,5 \mathrm{a}$ & $5,5 a b$ & $30,9 b$ & $4,2 \mathrm{~b}$ & $15,4 a b$ & $46,0 \mathrm{~b}$ & $32,1 \mathrm{a}$ \\
\hline & Braquiária & $5,6 a$ & $2,1 \mathrm{c}$ & $20,6 a b$ & $1,1 \mathrm{a}$ & $10,1 \mathrm{a}$ & $7,7 \mathrm{a}$ & $25,1 \mathrm{c}$ & $3,1 b$ & $18,3 a$ & $43,4 b c$ & $37,6 a$ \\
\hline & Pinus & $5,1 b$ & $1,1 d$ & $7,0 \mathrm{c}$ & $0,1 b$ & $4,0 \mathrm{~b}$ & $2,2 \mathrm{c}$ & $18,5 d$ & $2,9 b$ & $6,0 \mathrm{c}$ & $24,3 d$ & $23,4 b$ \\
\hline & Solo Exposto & $5,5 \mathrm{a}$ & $1,1 b$ & $5,1 b$ & $0,3 b$ & $4,8 b$ & $1,8 \mathrm{c}$ & $29,9 b$ & $7,9 \mathrm{a}$ & $6,5 c$ & $36,4 \mathrm{c}$ & $18,3 b$ \\
\hline \multirow[t]{5}{*}{ Prof } & $0,00-0,05 \mathrm{~m}$ & 5,4 & $4,0 a$ & $24,8 a$ & $1,2 \mathrm{a}$ & $10,3 a$ & $6,5 a$ & $31,9 a$ & 4,9 & $17,0 \mathrm{a}$ & $49,1 a$ & $33,3 a$ \\
\hline & $0,05-0,10 \mathrm{~m}$ & 5,2 & $2,9 \mathrm{~b}$ & $17,0 \mathrm{~b}$ & $0,8 \mathrm{ab}$ & $6,9 \mathrm{~b}$ & $4,4 a b$ & $31,1 \mathrm{ab}$ & 5,5 & $11,8 \mathrm{ab}$ & $42,6 a b$ & $27,5 \mathrm{ab}$ \\
\hline & $0,10-0,20 \mathrm{~m}$ & 5,3 & $2,4 \mathrm{bc}$ & $13,2 \mathrm{c}$ & $0,6 b c$ & $6,7 b$ & $3,6 \mathrm{~b}$ & $29,2 \mathrm{ab}$ & 5,9 & $10,6 b$ & $39,8 b$ & $25,9 \mathrm{ab}$ \\
\hline & $0,20-0,40 \mathrm{~m}$ & 5,3 & $2,1 \mathrm{~cd}$ & $11,0 \mathrm{~cd}$ & $0,5 b c$ & $5,6 b$ & $3,3 b$ & $28,3 b$ & 5,8 & $9,1 b$ & $37,1 b$ & $21,9 \mathrm{~b}$ \\
\hline & $0,40-0,60 \mathrm{~m}$ & 5,4 & $1,8 d$ & $9,4 \mathrm{~d}$ & $0,3 c$ & $5,1 b$ & $2,9 b$ & $28,1 b$ & 5,2 & $7,4 b$ & $35,4 b$ & $20,0 b$ \\
\hline CVeg & & 4,20 ** & $156,14^{\star *}$ & 94,68 ** & $17,76^{* *}$ & $10,76^{\star *}$ & $18,54^{\star *}$ & $103,83^{\star *}$ & $33,95^{\star \star}$ & $14,04^{\star *}$ & $35,54^{\star *}$ & $21,81^{* *}$ \\
\hline P rof & & $0,43^{\text {ns }}$ & $34,11^{\star *}$ & $44,62^{\star *}$ & 10,09 ** & 5,70 ** & $6,34^{\star *}$ & 3,30 * & $0,73^{\mathrm{ns}}$ & 6,31 ** & 8,26 ** & 7,50 ** \\
\hline CVeg $x$ Prof & & $0,23^{\mathrm{ns}}$ & $5,69 * *$ & $4,65^{\star \star}$ & $1,05^{\mathrm{ns}}$ & $1,16^{\mathrm{ns}}$ & $1,35^{\mathrm{ns}}$ & $7,32^{\star *}$ & $3,02^{* *}$ & $0,89^{\text {ns }}$ & $3,02^{\star *}$ & $0,70^{\text {ns }}$ \\
\hline CV (\%) & & 6,97 & 21,83 & 23,4 & 59,05 & 47,11 & 53,44 & 12,08 & 34,83 & 50,48 & 17,75 & 28,65 \\
\hline
\end{tabular}

* Médias seguidas de mesma letra na coluna não diferem pelo teste de Tukey a $5 \%$ de probabilidade. 
Tabela 2. Desdobramentos das interações significativas para fósforo $(\mathrm{P})$, matéria orgânica $(\mathrm{MO})$, acidez potencial $(\mathrm{H}+\mathrm{Al})$, alumínio trocável $(\mathrm{Al}+++)$ e capacidade de troca catiônica (CTC) em profundidades e por tratamentos*

\begin{tabular}{|c|c|c|c|c|c|c|}
\hline \multirow{2}{*}{ Variáveis } & \multirow{2}{*}{ Tratamento } & \multicolumn{5}{|c|}{ Profundidades (m) } \\
\hline & & $0-0,05$ & $0,05-0,10$ & $0,10-0,20$ & $0,20-0,40$ & $0,40-0,60$ \\
\hline \multirow{5}{*}{$\begin{array}{l}\text { P resina } \\
\left(\mathrm{mg} \mathrm{dm}^{-3}\right)\end{array}$} & Cerrado & $7 \mathrm{aA}$ & $6 a \mathrm{~B}$ & $5 \mathrm{aBC}$ & $4 a B C$ & $4 \mathrm{aC}$ \\
\hline & Regeneração & $6 \mathrm{aA}$ & $4 \mathrm{bB}$ & $3 b B$ & $2 \mathrm{bB}$ & $2 a b B$ \\
\hline & Braquiária & $4 \mathrm{bA}$ & $2 \mathrm{bcAB}$ & $2 \mathrm{bAB}$ & $1 b B$ & $1 \mathrm{bB}$ \\
\hline & Pinus & $1 \mathrm{cA}$ & $1 \mathrm{cA}$ & $1 \mathrm{bA}$ & $1 \mathrm{bA}$ & $1 \mathrm{bA}$ \\
\hline & Solo Exposto & $1 \mathrm{cA}$ & $1 \mathrm{cA}$ & $1 \mathrm{bA}$ & $1 \mathrm{bA}$ & $1 \mathrm{bA}$ \\
\hline \multirow{5}{*}{$\begin{array}{l}\text { MO } \\
\left(\mathrm{g} \mathrm{dm}{ }^{-3}\right)\end{array}$} & Cerrado & $40,0 \mathrm{aA}$ & $23,0 \mathrm{aB}$ & $15,0 \mathrm{abBC}$ & $12,0 \mathrm{bBC}$ & $8,0 \mathrm{bC}$ \\
\hline & Regeneração & $35,0 \mathrm{aA}$ & $24,3 \mathrm{aAB}$ & $21,3 \mathrm{aB}$ & $19,3 \mathrm{aB}$ & $21,0 \mathrm{aB}$ \\
\hline & Braquiária & $31,0 \mathrm{aA}$ & $24,3 \mathrm{aAB}$ & $19,3 \mathrm{aBC}$ & $16,0 \mathrm{abBC}$ & $12,6 \mathrm{abC}$ \\
\hline & Pinus & $8,3 \mathrm{bA}$ & $6,3 \mathrm{bA}$ & $6,3 \mathrm{bA}$ & $5,0 \mathrm{bA}$ & $4,0 \mathrm{bA}$ \\
\hline & Solo Exposto & $10,0 \mathrm{bA}$ & $7,0 \mathrm{bAB}$ & $4,0 \mathrm{bA}$ & $3,0 \mathrm{bA}$ & $2,0 \mathrm{bA}$ \\
\hline \multirow{5}{*}{$\begin{array}{l}\mathrm{H}+\mathrm{Al} \\
\left(\mathrm{mmol}_{\mathrm{c}} \mathrm{dm}^{-3}\right)\end{array}$} & Cerrado & $57,0 \mathrm{aA}$ & $55,0 \mathrm{aA}$ & $41,0 \mathrm{aB}$ & $35,0 \mathrm{aB}$ & $32,0 \mathrm{aB}$ \\
\hline & Regeneração & $29,0 \mathrm{bA}$ & $29,0 \mathrm{bA}$ & $31,0 \mathrm{aA}$ & $32,0 \mathrm{aA}$ & $33,0 \mathrm{aA}$ \\
\hline & Braquiária & $26,0 \mathrm{bA}$ & $26,0 \mathrm{bA}$ & $26,0 \mathrm{bA}$ & $24,0 \mathrm{abA}$ & $25,0 \mathrm{abA}$ \\
\hline & Pinus & $19,0 \mathrm{bA}$ & $18,0 \mathrm{bA}$ & $18,0 \mathrm{bA}$ & $19,0 \mathrm{bA}$ & $18,0 \mathrm{bA}$ \\
\hline & Solo Exposto & $29,0 \mathrm{bA}$ & $28,0 \mathrm{bA}$ & $29,0 \mathrm{bA}$ & $31,0 \mathrm{aA}$ & $33,0 \mathrm{aA}$ \\
\hline \multirow{5}{*}{$\begin{array}{l}\mathrm{Al}^{+++} \\
\left(\mathrm{mmol}_{\mathrm{c}} \mathrm{dm}^{-3}\right)\end{array}$} & Cerrado & $9 a A B$ & $12 \mathrm{aA}$ & $11 \mathrm{aA}$ & $8 \mathrm{aAB}$ & $5 a B$ \\
\hline & Regeneração & $1 \mathrm{bA}$ & $3 \mathrm{bA}$ & $5 a A$ & $6 a A$ & $6 a b A$ \\
\hline & Braquiária & $4 a A$ & $3 b A$ & $3 b A$ & $3 a A$ & $2 \mathrm{bA}$ \\
\hline & Solo Exposto & $7 a b A$ & $7 a b A$ & $7 a b A$ & $8 \mathrm{aA}$ & $10 \mathrm{aA}$ \\
\hline & Pinus & $3 b A$ & $2 \mathrm{bA}$ & $3 b A$ & $3 a A$ & $3 \mathrm{bA}$ \\
\hline \multirow{5}{*}{$\begin{array}{l}\text { CTC } \\
\left(\mathrm{mmol}_{\mathrm{c}} \mathrm{dm}^{-3}\right)\end{array}$} & Cerrado & $78,1 \mathrm{aA}$ & $64,8 \mathrm{aAB}$ & 48,9abB & $40,9 \mathrm{aB}$ & $36,6 a \mathrm{~B}$ \\
\hline & Regeneração & $54,9 \mathrm{bA}$ & $43,7 \mathrm{aA}$ & $44,6 \mathrm{abA}$ & $44,2 \mathrm{aA}$ & $42,6 a A$ \\
\hline & Braquiária & $50,2 \mathrm{bA}$ & $45,7 \mathrm{abA}$ & $43,7 \mathrm{abA}$ & $40,6 \mathrm{aA}$ & $36,7 \mathrm{aA}$ \\
\hline & Pinus & $26,3 \mathrm{cA}$ & $23,6 \mathrm{bA}$ & $25,8 \mathrm{bA}$ & $23,4 \mathrm{aA}$ & $22,7 \mathrm{aA}$ \\
\hline & Solo Exposto & $35,6 \mathrm{bcA}$ & $35,1 \mathrm{bA}$ & $35,9 \mathrm{bA}$ & $36,5 \mathrm{aA}$ & $38,8 \mathrm{aA}$ \\
\hline
\end{tabular}

* Médias seguidas de mesma letra, minúscula na coluna e maiúscula na linha, para cada variável, não diferem pelo teste de Tukey a $5 \%$ de probabilidade

que, a partir desta profundidade, a CTC deixa de ser influenciada pelos conteúdos de MO e indicando a presença de mesmo substrato inorgânico (Reichmann Neto \& Santos Filho, 1982), que pode desempenhar importante papel no incremento da CTC em solos bastante intemperizados.

Tanto para MO como para CTC, o subsolo, em processo de regeneração natural e com braquiária, apresenta incrementos que apontam no sentido da recuperação desses atributos que, de modo comparativo, seriam considerados recuperados quando nenhuma diferença significativa fosse verificada entre o cerrado natural e as áreas em análise.

Assim como a MO e a CTC, a soma de bases (SB) e a saturação por bases (V\%) decresceram em profundidade e se mostraram com menores valores nos tratamentos cerrado, Pinus e solo exposto (Tabela 1). Por se tratar, o subsolo em estudo, de material quimicamente pobre, nota-se que a maior parte dos nutrientes é proveniente da vegetação e está relacionada aos conteúdos de MO, como verificado também por Garay et al. (2003).

O comportamento diagnosticado sugere, mais uma vez, que a braquiária e a regeneração produziram melhores resultados químicos na recuperação da área em foco.

Comportamento semelhante também foi verificado para o pH (Tabela 1), que tem no tratamento Pinus o menor valor. $\mathrm{O}$ pH não apresentou diferenças significativas em profundidade sendo classificado, portanto, como moderadamente ácido $(5,5$ a 5,8) para a braquiária e fortemente ácido $(4,9$ a 5,3) para os demais tratamentos (EMBRAPA, 1999).
Ao se avaliar a acidez potencial $(\mathrm{H}+\mathrm{Al})$ em profundidade, dentro de cada tratamento (Tabela 2), nota-se que o cerrado se destacou por apresentar maior acidez até os $0,10 \mathrm{~m}$, diferindo significativamente dos demais tratamentos. Nas outras profundidades, a regeneração não diferiu do Cerrado. O Pinus foi homogêneo em profundidade e apresentou o menor valor de acidez potencial, embora conjuntamente com regeneração, braquiária e solo exposto, se mostraram homogêneos entre si e em profundidade.

A presença de fósforo nas áreas em estudo (Tabela 2) é incipiente, podendo ser considerada de teores medianos nos primeiros centímetros do cerrado e da regeneração apresentando, no Pinus e no solo exposto, seus menores valores.

\section{Atributos físicos}

A densidade de partículas (Tabela 3) pode auxiliar na identificação dos minerais presentes no material de solo (Kiehl, 1979). Tem-se nos solos da região, como minerais predominantes, caulinita e quartzo (Demattê, 1980; IPT, 1981; Kiehl, 1979; Maltoni, 1994; Moniz \& Carvalho, 1973), com densidade de partículas média de 2,65 $\mathrm{kg} \mathrm{dm}^{-3}$, além de hematita e magnetita com densidade de partículas média de 5,00 $\mathrm{kg} \mathrm{dm}^{-3}$ gerando densidades da ordem de 2,5 a $2,7 \mathrm{~kg} \mathrm{dm}^{-3}$. Em associação com essas informações, os dados obtidos no presente trabalho indicam ausência de variações significativas em profundidade, indicando homogeneidade de material. Entre os tratamentos as diferenças encontradas podem estar indicando 
Tabela 3. Médias* e valores de F para macroporos, microporos, porosidade total, densidade de partículas e densidade do solo, nas diferentes coberturas vegetais (CVeg) e profundidades (Prof)

\begin{tabular}{|c|c|c|c|c|c|c|}
\hline \multirow{2}{*}{ Variáveis } & \multirow{2}{*}{ Tratamentos } & Macroporos & \multirow{2}{*}{$\begin{array}{c}\text { Microporos } \\
\left(\mathrm{m}^{3} \mathrm{~m}^{-3}\right)\end{array}$} & \multirow[t]{2}{*}{ Porosidade Total } & \multicolumn{2}{|c|}{ Densidade $-\mathrm{kg} \mathrm{dm}^{-3}$} \\
\hline & & & & & Partícula & Solo \\
\hline \multirow[t]{5}{*}{ CVeg } & Cerrado & $0,24 a$ & $0,26 \mathrm{~b}$ & $0,50 \mathrm{a}$ & $2,73 b$ & $1,24 \mathrm{a}$ \\
\hline & Regeneração & $0,11 b c$ & $0,27 \mathrm{~b}$ & $0,38 \mathrm{~cd}$ & $2,74 a b$ & $1,60 \mathrm{~b}$ \\
\hline & Braquiária & $0,13 b$ & $0,27 \mathrm{~b}$ & $0,41 \mathrm{c}$ & $2,70 \mathrm{~b}$ & $1,50 \mathrm{~b}$ \\
\hline & Pinus & $0,11 b c$ & $0,25 b$ & $0,35 d$ & $2,72 b$ & $1,70 \mathrm{c}$ \\
\hline & Solo Exposto & $0,09 c$ & $0,35 a$ & $0,46 b$ & $2,80 \mathrm{a}$ & $1,53 b$ \\
\hline \multirow[t]{4}{*}{ Prof } & $0,00-0,10 \mathrm{~m}$ & $0,15 a$ & 0,27 & 0,42 & 2,73 & 1,48 \\
\hline & $0,10-0,20 \mathrm{~m}$ & $0,15 a b$ & 0,27 & 0,42 & 2,75 & 1,50 \\
\hline & $0,20-0,40 \mathrm{~m}$ & $0,13 b c$ & 0,29 & 0,42 & 2,73 & 1,54 \\
\hline & $0,40-0,40 \mathrm{~m}$ & $0,12 \mathrm{c}$ & 0,30 & 0,42 & 2,75 & 1,54 \\
\hline CVeg & & $76,76^{*}$ & $23,47^{*}$ & $40,69 *$ & $5,79 *$ & $41,45^{*}$ \\
\hline Prof & & $6,27^{\star}$ & $2,75^{\mathrm{ns}}$ & $0,17^{\mathrm{ns}}$ & $0,76^{\mathrm{ns}}$ & $1,79^{\text {ns }}$ \\
\hline CVeg x Prof & & $2,64^{* *}$ & $0,66^{\mathrm{ns}}$ & $1,95^{\mathrm{ns}}$ & $0,49^{\text {ns }}$ & 2,94 * \\
\hline CV (\%) & & 17,15 & 9,74 & 8,16 & 1,88 & 6,08 \\
\hline
\end{tabular}

* Médias seguidas de mesma letra na coluna não diferem pelo teste de Tukey a $5 \%$ de probabilidade.

diferentes profundidades de corte, o que aproximaria mais o subsolo do basalto, presente na região.

O comportamento da densidade do solo (Tabela 3), sugere que as coberturas vegetais presentes na área, com o objetivo de recuperá-las, apresentam algum efeito sobre a densidade do material, pelo menos nos primeiros $0,10 \mathrm{~m}$. É interessante observar que, em valores absolutos, a densidade das áreas sob Pinus é mais elevada que no material sob solo exposto e sob cerrado, fato que pode estar associado aos maiores conteúdos de areia presentes neste (Kiehl, 1979), como mostram os dados da Tabela 5.

Ressalta-se que valores de densidade do solo da ordem de

Tabela 4. Desdobramento das interações significativas para densidade do solo e macroporos, por tratamento*, em profundidade

\begin{tabular}{llllll}
\hline \multirow{2}{*}{ Variáveis } & Tratamentos & \multicolumn{4}{c}{ Profundidade $(\mathbf{m})$} \\
\cline { 3 - 6 } Densidade do Solo & Cerrado & $\mathbf{0 - 0 , 1 0}$ & $\mathbf{0 , 1 0 - 0 , 2 0}$ & $\mathbf{0 , 2 0 - 0 , 4 0}$ & $\mathbf{0 , 4 0 - 0 , 6 0}$ \\
$\left(\mathrm{kg} \mathrm{dm}^{-3}\right)$ & Regeneração & $1,47 \mathrm{abB}$ & $1,31 \mathrm{aA}$ & $1,26 \mathrm{aA}$ & $1,19 \mathrm{aA}$ \\
& Braquiária & $1,56 \mathrm{bB}$ & $1,48 \mathrm{abB}$ & $1,68 \mathrm{bcC}$ & $1,72 \mathrm{bcC}$ \\
& Pinus & $1,56 \mathrm{bB}$ & $1,64 \mathrm{bC}$ & $1,80 \mathrm{cC}$ & $1,50 \mathrm{bB}$ \\
& Solo Exposto & $1,59 \mathrm{bB}$ & $1,54 \mathrm{bBC}$ & $1,51 \mathrm{abcB}$ & $1,47 \mathrm{abB}$ \\
Macroporos $^{3}$ & Cerrado & $0,26 \mathrm{aA}$ & $0,23 \mathrm{aA}$ & $0,24 \mathrm{aA}$ & $0,24 \mathrm{aA}$ \\
$\left(\mathrm{m}^{3} \mathrm{~m}^{-3}\right)$ & Regeneração & $0,15 \mathrm{bA}$ & $0,14 \mathrm{bA}$ & $0,09 \mathrm{cA}$ & $0,07 \mathrm{bB}$ \\
& Braquiária & $0,11 \mathrm{bA}$ & $0,15 \mathrm{bA}$ & $0,15 \mathrm{bcA}$ & $0,12 \mathrm{bA}$ \\
& Pinus & $0,13 \mathrm{bA}$ & $0,11 \mathrm{bA}$ & $0,07 \mathrm{cA}$ & $0,07 \mathrm{bA}$ \\
& Solo Exposto & $0,11 \mathrm{bA}$ & $0,10 \mathrm{bA}$ & $0,10 \mathrm{cA}$ & $0,11 \mathrm{bA}$ \\
\hline
\end{tabular}

* Médias seguidas de mesma letra, minúscula na coluna e maiúscula na linha, não diferem pelo teste de Tukey a $5 \%$ de probabilidade.

Tabela 5. Resultado da análise granulométrica e classe textural

\begin{tabular}{lcccc}
\hline Tratamentos & Argila & Silte & Areia & $\begin{array}{c}\text { Classe } \\
\text { Textural }\end{array}$ \\
\cline { 2 - 3 } Cerrado & & $\mathbf{g ~ k g}^{-1}$ & & argilosa \\
Regeneração & 385 & 185 & 430 & média \\
Braquiária & 296 & 159 & 545 & média \\
Pinus & 306 & 119 & 575 & média \\
Solo Exposto & 207 & 161 & 632 & média \\
\hline
\end{tabular}

1,6 kg dm³ ração (Tabela 4), podem comprometer o desenvolvimento do sistema radicular das vegetações introduzidas uma vez que densidades na faixa de 1,6 a $1,8 \mathrm{~kg} \mathrm{dm}^{-3}$, como relatado por Corrêa \& Melo (1998), impedem o desenvolvimento de raízes.

A porosidade total não apresentou variações significativas em profundidade, sendo considerada homogênea (Tabela 3). No entanto, a macroporosidade foi superior no cerrado (Tabela 4) explicando o comportamento da velocidade de infiltração da água (Figura 1), na qual regeneração, Pinus, braquiária e solo exposto se diferenciam do cerrado e apresentam as menores velocidades de infiltração de água, devido a menor macroporosidade e maior densidade do solo, observado também por Anjos et al. (1994) e Corrêa \& Melo (1998).

Schneider et al. (2003) relatam que baixas taxas de infiltração de água no solo ocorrem onde há redução no volume de poros, especialmente de macroporos, o que pode implicar em incrementos na resistência do solo à penetração de raízes.

A diferença entre a velocidade de infiltração da água na área de cerrado e nas demais foi bastante grande (Figura 1), indicando haver problemas relativos a infiltração de água e, conseqüentemente, maior risco de erosão (observada em campo), em conseqüência da baixa velocidade de infiltração.

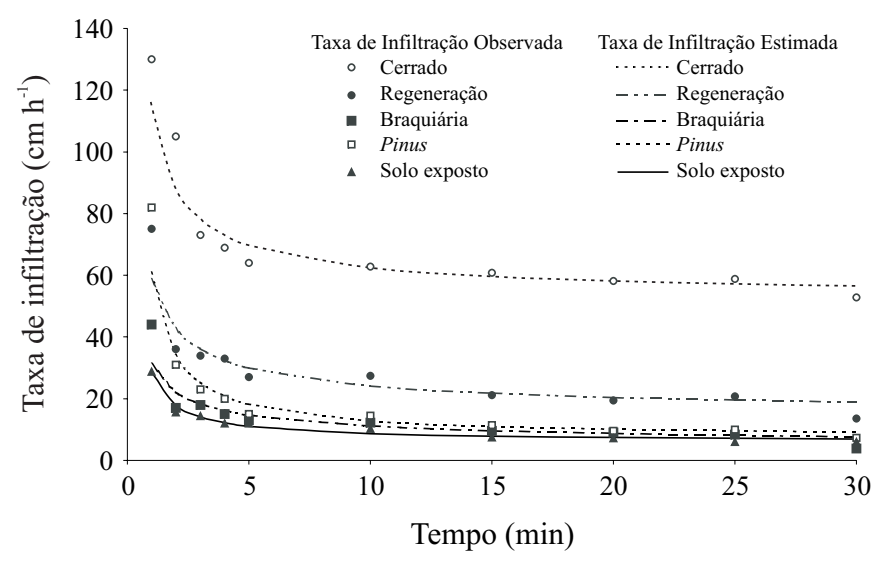

Figura 1. Taxa de infiltração de água, em função dos tratamentos 
Devido ao escorrimento superficial gerado, na maioria das vezes, pela lentidão com que a água se infiltra no solo, a produção de sedimentos poderá contribuir para o assoreamento de cursos d'água e comprometimento da sua qualidade.

Além da densidade, da porosidade, macroporosidade e microporosidade, a morfologia e a arquitetura dos poros são muito importantes na condução da água em profundidade, assim como a presença de compactação superficial, que poderá dificultar este processo (Carnicelli et al., 1994; Hallaire \& Curmi, 1994).

Os maiores valores de diâmetros médios ponderados (DMP) e geométricos (DMG) (Tabela 6) foram encontrados no cerrado e na braquiária, nas duas profundidades analisadas (0-0,10 e 0,10-0,20 m), dados coincidentes com os maiores percentuais de agregados (> 2,0 mm de diâmetro) encontrados nesses tratamentos (Tabela 6) situação em que, segundo Angulo et al. (1984), se teria a melhor taxa de infiltração de água no solo; este efeito não se faz claro nos tratamentos presentes em que a maior velocidade de infiltração, depois do cerrado, ocorreu na regeneração (Figura 1).

Além dessas variáveis, deve-se considerar, para esta avaliação, a possibilidade de condução de água pelos poros e a presença de encrostamento superficial. Segundo Castro Filho et al. (1998), a manutenção da arquitetura dos poros pela permanência dos restos de raízes, ação da macro e microfauna na fragmentação desses resíduos e formação de galerias, influi na aeração e na movimentação descendente de água, produzindo trocas mais intensas e contribuindo para melhorar a agregação, o que pode estar ocorrendo com mais intensidade na área em regeneração que nas demais áreas.

Além da estrutura e da velocidade de infiltração, tem-se a temperatura como um dos fatores importantes para o desenvolvimento da vegetação, uma vez que afeta os processos físicos, químicos e biológicos imprescindíveis para seu bom desenvolvimento (Entry et al., 2002). A decomposição da matéria orgânica também é afetada pela temperatura do solo, dentre outros fatores climáticos e edáficos (Silva \& Resck, 1997).

A presença de cobertura vegetal ou de seus resíduos, na superfície do solo, intercepta a radiação solar e influencia no balanço de energia solar (Pereira et al., 2002). Em uma
Tabela 7. Valores médios* para temperatura $\left({ }^{\circ} \mathrm{C}\right)$ ao longo do ano no período da manhã e da tarde

\begin{tabular}{llll}
\hline \multirow{2}{*}{ Tratamentos } & Manhã & Tarde & $\Delta$ \\
\cline { 2 - 4 } Cerrado & \multicolumn{3}{c}{$\boldsymbol{\Delta}$} \\
Regeneração & $22,1 \mathrm{aA}$ & $24,7 \mathrm{aB}$ & 2,6 \\
Braquiária & $23,3 \mathrm{abA}$ & $29,7 \mathrm{bB}$ & 6,4 \\
Pinus & $24,4 \mathrm{bA}$ & $33,4 \mathrm{cB}$ & 9,0 \\
Solo Exposto & $23,1 \mathrm{abA}$ & $30,6 \mathrm{bB}$ & 7,5 \\
\hline
\end{tabular}

* Médias seguidas de mesma letra, minúscula na coluna e maiúscula na linha não diferem pelo teste de Tukey a $5 \%$ de probabilidade

$\Delta=$ temperatura da tarde menos a temperatura da manhã, isto é, variação de temperatura ocorrida entre as medidas realizadas.

tentativa de se avaliar o recobrimento do solo pela vegetação, mediu-se a temperatura (Tabela 7). As menores variações foram encontradas sob cerrado onde a vegetação é mais exuberante e recobre o solo ao longo do dia e do ano, sendo seguida pela regeneração e pelo Pinus, que apresentam bom recobrimento, quando comparados com braquiária e solo exposto. Nesses tratamentos (braquiária e solo exposto) as variações de temperatura a 0,04 $\mathrm{m}$ de profundidade, passam da casa dos $10{ }^{\circ} \mathrm{C}$ em pelo menos um dos meses avaliados e podem influenciar o comportamento dos fungos e da fauna do solo, como observado por Doran \& Parkin (1994); Entry et al. (2002) e Hungria et al. (1997).

\section{CONCLUSÕES}

1. As coberturas vegetais utilizadas na revegetação do subsolo estudado estão modificando as suas propriedades físicas e químicas.

2. O Pinus não foi uma boa espécie para recuperar os atributos químicos do material, na região da UHE de Ilha Solteira, em Selvíria, MS.

3. As áreas revegetadas precisam ser melhoradas em seus atributos físicos, podendo haver adequado desenvolvimento da vegetação.

4. A braquiária e a regeneração apresentaram resultados melhores e se aproximaram mais do cerrado natural.

Tabela 6. Diâmetro médio* ponderado (DMP), diâmetro médio geométrico (DMG) e distribuição dos agregados (em porcentagem) por classe de diâmetro (mm), em função dos tratamentos e das profundidades

\begin{tabular}{|c|c|c|c|c|c|c|c|}
\hline \multirow{2}{*}{ Tratamentos } & DMP & DMG & $>2$ & $2-1$ & $1-0,5$ & $0,5-0,25$ & $<0,25$ \\
\hline & \multicolumn{2}{|c|}{$\mathrm{mm}$} & \multicolumn{5}{|c|}{$\%$} \\
\hline & \multicolumn{7}{|c|}{ Prof $0-0,10 \mathrm{~m}$} \\
\hline Cerrado & $2,83 \mathrm{aA}$ & $1,57 \mathrm{aA}$ & $93,20 \mathrm{aA}$ & 1,55aA & $0,64 \mathrm{aA}$ & $0,75 \mathrm{aA}$ & $3,86 \mathrm{aA}$ \\
\hline Regeneração & $2,47 a b A$ & $1,37 \mathrm{bA}$ & $76,60 \mathrm{abA}$ & $6,34 a b A$ & $4,24 \mathrm{aA}$ & $5,51 \mathrm{bA}$ & $7,27 \mathrm{bA}$ \\
\hline Braquiária & $2,70 \mathrm{abA}$ & $1,51 \mathrm{abA}$ & $87,30 \mathrm{abA}$ & 3,57abA & $1,65 \mathrm{aA}$ & $1,81 \mathrm{abA}$ & $5,71 \mathrm{abA}$ \\
\hline Pinus & $2,38 \mathrm{bA}$ & $1,37 \mathrm{bA}$ & $71,90 \mathrm{bA}$ & $10,51 \mathrm{bA}$ & $5,59 \mathrm{aA}$ & $4,73 a b A$ & $7,30 \mathrm{bA}$ \\
\hline \multirow[t]{2}{*}{ Solo Exposto } & $2,60 \mathrm{abA}$ & $1,44 a b A$ & $82,00 \mathrm{abA}$ & $5,57 \mathrm{abA}$ & $4,17 \mathrm{aA}$ & $3,81 \mathrm{abA}$ & $4,45 a b A$ \\
\hline & \multicolumn{7}{|c|}{ Prof $0,10-0,20 \mathrm{~m}$} \\
\hline Cerrado & $2,81 \mathrm{aA}$ & $1,57 \mathrm{aA}$ & $91,60 \mathrm{aA}$ & 2,31aA & $0,91 \mathrm{aA}$ & $0,84 \mathrm{aA}$ & $4,34 \mathrm{aA}$ \\
\hline Regeneração & $2,39 a b A$ & $1,36 \mathrm{bA}$ & $73,60 \mathrm{bA}$ & $9,66 a b c A$ & $6,21 \mathrm{bA}$ & $5,89 \mathrm{bA}$ & 4,64abA \\
\hline Braquiária & 2,64abA & $1,47 a b c A$ & $84,47 a b A$ & 4,18abA & $2,65 \mathrm{abA}$ & $3,84 a b A$ & $4,86 a b A$ \\
\hline Pinus & $2,30 \mathrm{bA}$ & $1,32 \mathrm{bA}$ & $68,34 \mathrm{bA}$ & $10,23 \mathrm{bcA}$ & $7,52 \mathrm{bA}$ & $6,47 \mathrm{bA}$ & $7,44 \mathrm{bA}$ \\
\hline Solo Exposto & $1,81 \mathrm{cB}$ & $1,16 \mathrm{cB}$ & $49,75 \mathrm{cB}$ & $13,70 \mathrm{cB}$ & $12,67 \mathrm{cB}$ & $12,39 \mathrm{cB}$ & $11,49 \mathrm{cB}$ \\
\hline
\end{tabular}

* Médias seguidas de mesma letra, minúscula na coluna e por profundidade, maiúscula na coluna, por tratamento e entre profundidades, não diferem pelo teste de Tukey a $5 \%$ de probabilidade 


\section{AGRADECIMENTOS}

Os autores manifestam seu agradecimento ao Professor Dr. Gilson Volpato, da UNESP, pelas sugestões para a redação científica; ao Sr. João Josué Barbosa, pelo auxílio na normatização bibliográfica e ao Professor Dr. Paulo C. Ceresini, pela revisão do Abstract.

\section{LITERATURA CITADA}

Almeida, R. O. P. O. Revegetação de áreas mineradas: estudo dos procedimentos aplicados em minerações de areia. São Paulo: USP, 2002. 160p. Dissertação Mestrado

Angulo, R. J.; Roloff, G.; Souza, M. L. P. Relações entre a erodibilidade e agregação, granulometria e características químicas de solos brasileiros. Revista Brasileira de Ciência do Solo, Viçosa, v.8, n.1, p.133-138, 1984.

Anjos, J. T.; Uberti, A. A. A.; Vizzotto, V. J.; Leite, G. B.; Krieger, M. Propriedades físicas em solos sob diferentes sistemas de uso e manejo. Revista Brasileira de Ciência do Solo, Viçosa, v.18, n.1, p.139-145, 1994.

Carnicelli, S.; Ferrari, G. A.; Pagliai, M. Pore space degradation in Zimbabwean crusting soils. In: Rindrose-Voase, A. J.; Humphreys, G. S. (ed.). Soil micromorphology: studies in management and genesis. Amsterdam: Elsevier, 1994. p.677-687.

Castro Filho, C.; Muzilli, O.; Podanoschi, A. Estabilidade dos agregados e sua relação com o teor de carbono orgânico num Latossolo Roxo Distrófico, em função de sistemas de plantio, rotações de culturas e métodos de preparo das amostras. Revista Brasileira de Ciência do Solo, Viçosa, v.22, n.3, p.527-538, 1998.

Corrêa, R. S.; Melo, B. F. Ecologia da revegetação em áreas escavadas. In: Corrêa, R. S.; Melo, B. F. (ed.). Ecologia e recuperação de áreas degradadas no Cerrado. Brasília: Paralelo 15, 1998. p.65-99.

Demattê, J. L. I. Levantamento detalhado dos solos do "Campus experimental de Ilha Solteira”. Piracicaba: ESALQ, 1980. 119p.

Dias Filho, M. B. Pastagens cultivadas na Amazônia oriental brasileira: processos e causas de degradação e estratégias de recuperação. In: Dias, L. E.; Mello, J. W. V. (ed.). Recuperação de áreas degradadas. Viçosa: Sociedade Brasileira de Recuperação de Áreas Degradadas, 1998. p.135-147.

Dias, L. E.; Griffith J. J. Conceituação e caracterização de áreas degradadas. In: Dias, E. L.; Mello, J. W. V. (ed.). Recuperação de áreas degradadas. Viçosa: UFV/Departamento de Solos; Sociedade Brasileira de Recuperação de Áreas Degradadas, 1998. p.1-7.

Doran, J. W.; Parkin, T. B. Defining and assessing soil quality. In: Doran, J. M.; Coleman, D. C.; Bezdicek, D. F.; Stewart, B. A. (ed.). Defining soil quality for a sustainable environment. Madison: Soil Science Society of America, 1994. p.3-21. Special Publication, 35.

Durigan, G. Técnicas silviculturais à restauração. In: Simpósio sobre Restauração Ecológica de Ecossistemas Naturais, 1, 1999, Piracicaba. Anais... Piracicaba: IPEF, 1999. CD-Rom.
EMBRAPA - Empresa Brasileira de Pesquisa Agropecuária. Centro Nacional de Pesquisa de Solos. Manual de métodos de análise de solo. 2.ed. Rio de Janeiro: CNPS, 1997. 212p.

EMBRAPA - Empresa Brasileira de Pesquisa Agropecuária. Sistema brasileiro de classificação de solos. Rio de Janeiro:CNPS, 1999. 412p.

Entry, J. A.; Rygiewicz, P. T.; Watrud, L. S.; Donnelly, P. K. Influence of adverse soil conditions on the formation and function of Arbuscular mycorrhizas. Advances in Environmental Reserch, Amsterdam, v.7, n.1, p.123-138, 2002.

Garay, I.; Kindel, A.; Carneiro, R.; Franco, A. A.; Barros, E.; Abbadie, L. Comparação da matéria orgânica e de outros atributos do solo entre plantações de Acacia mangium e Eucalyptus grandis. Revista Brasileira de Ciência do Solo, Viçosa, v.27, n.4, p.705-712, 2003.

Hallaire, V.; Curmi, P. Image analysis of pore space morphology in soil sections, in relation to water movement. In: RindroseVoase, A. J.; Humphreys, G. S. (ed.). Soil micromorphology: Studies in management and genesis. Amsterdam: Elsevier, 1994. p.559-567.

Hungria, M.; Vargas, M. A. T.; Araújo, R. S. Fixação biológica do nitrogênio em feijoeiro. In: Vargas, M. A. T.; Hungria, M. (ed.). Biologia dos solos dos cerrados. Planaltina: Embrapa-CPAC, 1997. p.187-258.

IPT - Instituto de Pesquisas Tecnológicas do Estado de São Paulo. Mapa geológico do Estado de São Paulo: Escala 1:500000. v.I e II. São Paulo: IPT, 1981. 126p. Monografias, 6.

Kiehl, E. J. Manual de edafologia: relação solo-planta. São Paulo: Agronômica Ceres, 1979. 264p.

Lyle, E. Surface mine reclamation manual. New York: Elsevier, 1987. p.62-94.

Maltoni, K. L. Estudo de compactação e/ou adensamento em superfície de Latossolo sob diferentes usos. Viçosa: UFV, 1994. 139p. Tese Doutorado.

Moniz, A. C.; Carvalho, A. Seqüência de evolução de solos derivados do arenito Bauru e de rochas básicas da região noroeste do estado de São Paulo. Bragantia: Campinas, v.32, n.17, p.309-333, 1973.

Moreira, P. R. Manejo do solo e recomposição da vegetação com vistas a recuperação de áreas degradadas pela extração de bauxita, Poços de Caldas, MG. Rio Claro: UNESP, 2004. 139p. Tese Doutorado

Pereira, A. R.; Angelocci, L. R.; Sentelhas, P. C. Agrometeorologia: fundamentos e aplicações práticas. Guaíba: Agropecuária, 2002. 478p.

Prandini, F. L.; Iwasa, O. Y.; Oliveira, A. M. S. A cobertura vegetal nos processos e evolução do relevo: o papel da floresta. In: Congresso Nacional sobre Essencias Nativas, 1, 1982, Campos de Jordão. Anais... Campos de Jordão: Silvicultura em São Paulo, 1982. p.1568-1582.

Reichmann Neto, F.; Santos Filho, A. Desenvolvimento de solos em "áreas de empréstimo", resultante do plantio de Gramíneas e Bracatinga. In: Congresso Nacional sobre Essencias Nativas, 1, 1982, Campos de Jordão. Anais... Campos de Jordão: Silvicultura em São Paulo, 1982. p.1896-1899.

Resende, M.; Curi, N; Rezende, S. B.; Côrrea, G. F. Pedologia: base para distinção de ambientes. 4.ed. Viçosa: Neput, 2002. p.237-257. 
Sánchez, P. A. Suelos del tropico: caracteristicas y manejo. San José: IICA, 1981. 660p.

SAS - Statistical Analysis System Institute - SAS/STAT Procedure guide for personal computers. 5.ed. Cary, NC: SAS Inst. 1999. 334p.

Schneider, J.; Soler, M. A.; Baretta, D.; Leite, D.; Mafra, A. L.; Nagaoka, A. N. Taxa de infiltração e resistência ao penetrômetro em semeadura direita com diferentes profundidades de sulcamento. In: Congresso Brasileiro de Ciência do Solo, 29, 2003, Ribeirão Preto. Anais... Ribeirão Preto: UNESP, 2003. CD-Rom.
Silva, J. E.; Resck, D. V. S. Matéria orgânica do solo. In: Vargas, M. A. T.; Hungria, M. (eds.). Biologia dos solos dos cerrados. Planaltina: Embrapa-CPAC, 1997. p.465-516.

Sposito, G. The aims of soil science-challenges to be taken up by soil science - The aplications and benefits of soil science. In: World Congress of Soil Science, 16, Montpellier-França. Anais... Montpellier:International Society of Soil Science, 1998. p.41-48.

van Raij, B.; Quaggio, J. A. Métodos de análise de solo para fins de fertilidade. Campinas: Instituto Agronômico, 1983. 31p. Boletim Técnico, 81. 